\title{
LMC OB associations containing Wolf-Rayet stars
}

\author{
H. Schild \\ Department of Physics and Astronomy \\ University College London \\ Gower Street, London WC1E 6BT \\ England
}

\author{
M.C. Lortet and G. Testor \\ Observatoire de Paris \\ Section de Meudon/DAEC \\ F-92195 Meudon Principal Cedex \\ France
}

\begin{abstract}
.
We observed four OB associations in the Large Magellanic Cloud which contain Wolf-Rayet stars. The associations are located in the neighbourhood of 30 Dor. They contain 15 WR stars of WCE and most WN subtypes. The observations include UBV photometry for several thousand objects and spectroscopy in the range from 3500 to $5000 \AA$ for about 250 stars. Two new extreme Of stars and a new WR star as well as many hitherto unknown $\mathrm{O}$ stars were identified. Cluster HR diagrams reveal the age structure of the association stars and yield tentative conclusions about evolutionary connections between Wolf-Rayet stars in the LMC.
\end{abstract}

\section{Introduction}

The coincidence of Wolf-Rayet stars with OB associations provides an opportunity to determine ages of WR stars. If we assume that WR stars are the end product of standard stellar evolution, we can also in principal assign an initial mass to the WR progenitors. In the case of galactic WR stars, this has been done by Schild and Maeder (1984) and Humphreys et al. (1985).

The Wolf-Rayet stars in the LMC also have a high coincidence rate with $O B$ associations (e.g. Breysacher 1986). The stellar content of these associations is however very poorly known. Up to now, the only association with a WR star examined in detail is LH 39 which contains the WN9-10 star Brey 18 (Schild 1987). We therefore embarked on an observing program with the aim to determine the stellar content of some selected LMC OB associations. In a fist step we focussed on associations near 30 Dor.

\section{Observations}

During two observing runs in Dec 1988 and Dec 1989/Jan 1990 with the ESO $3.6 \mathrm{~m}$ telescope and the multi-purpose instrument EFOSC, we obtained spectra of about 250 and UBV colours of several thousand stars in the associations LH 89, LH 90, LH 99 and LH 104.

The CCD field of view was 3.' 6 x 5.'6. The photometric observations were reduced with DAOPHOT. It was possible to resolve many tight clusters. We estimate the internal photometric errors to be between 0.02 and $0.05 \mathrm{mag}$ for the bright $\left(m_{V}=12\right)$ and faint $\left(m_{V}=20\right)$ stars respectively. The main error source in the Johnson magnitudes comes from the scarcity of suitable calibration stars in the CCD fields.

The spectroscopic observations covered the range from 3900 to $5100 \AA$ with a resolution of $7 \AA$. The EFOSC instrument was used in its multi-object mode through aperture 
holes with a diameter of 2.1 arcsec. The data were reduced in a standard way by using STARLINK software available at UCL. Nebular HII emission lines present in many stellar spectra were usually not removed except in cases where spectral classification lines like HeI $\lambda 4471$ were affected.

\section{New Wolf-Rayet and extreme Of stars}

Our spectra revealed the presence of a hitherto unknown WR star as well as an extreme Of star in a tight cluster of LH 90 (Fig. 1). Brey 65 and star 1 are Wolf-Rayet stars of WN7 and WN3 type respectively, whereas star 2 is an extreme Of star. All three stars are of about the same $m_{V} \approx 12.9 \mathrm{mag}$ (Testor and Schild 1990). The fact that an early and late WN star are only separated by 3 arcsec may explain the different spectral types previously attributed to Brey 65: OB + WN (Smith 1968), WN3-5 (Fehrenbach et al. 1976), O: + WN5-6 (Walborn 1977) and WN4.5 + OB (Breysacher 1981), WN/Of? (Torres-Dodgen and Massey 1988), WN7 + O (Moffat 1989).

Fig. 1: B filter image of the central part of the OB association LH 90 with the WR and extreme Of stars. North is at the top, East to the left.

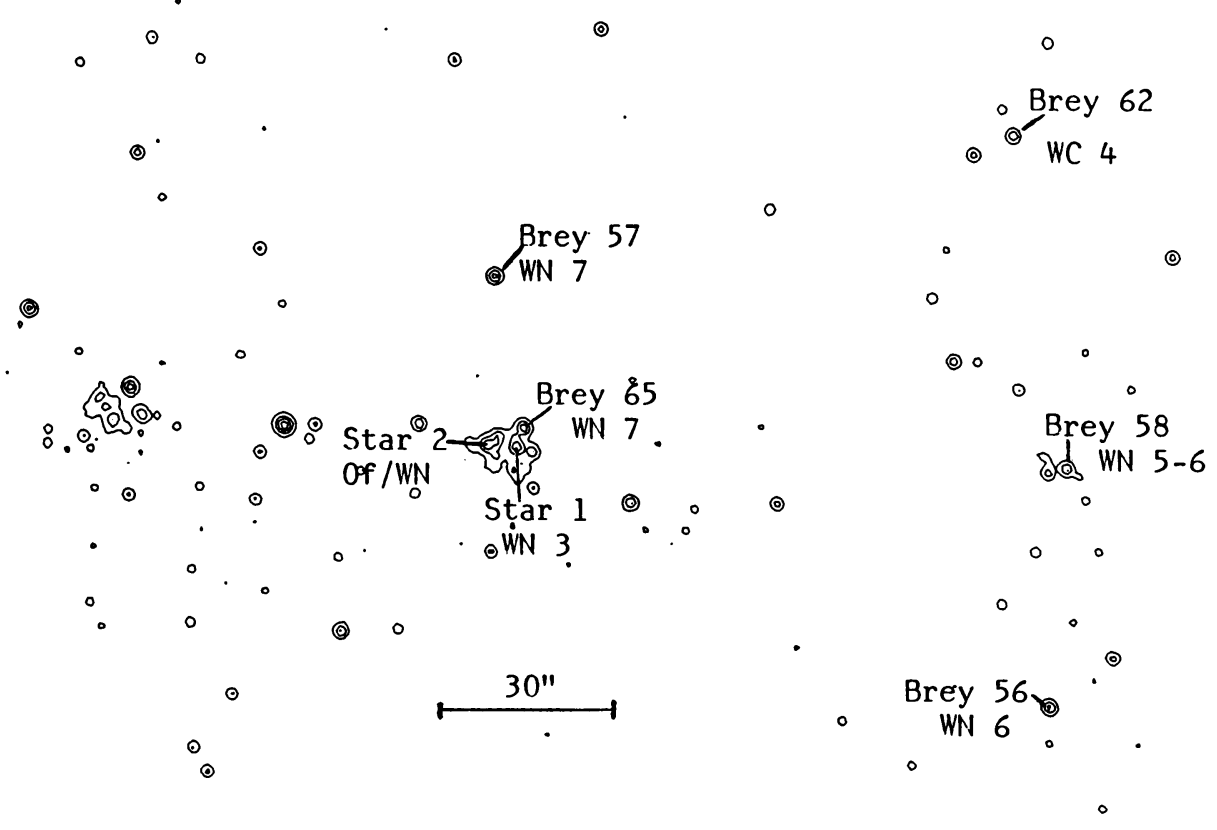

A further extreme Of star was found in a bright nebular region of LH 99 (for identification see Testor and Schild 1990). The star is of visual magnitude 13.6 and has a strong and broad HeII $\lambda 4686$ emission line. According to the classification criteria of Walborn (1986) it is of O3If/WN6 spectral type. 


\section{Stellar content of $O B$ associations}

\subsection{LH 99}

The spectroscopically surveyed area contains the three WR stars Brey 71, MGWR 4 and Brey 73 which are of WN7, WN3-4 and WN7 subtype, respectively. In addition, we find an O3If/WN6 transition object about 30" to the SE of Brey 73 (Testor and Schild 1990). LH 99 certainly is a very young stellar association. We find almost $40 \mathrm{O}$ stars, half a dozen of which are earlier than 05 . The HR diagram shows a well populated main sequence going up to $M_{b o l} \approx-10$ (Fig. 2). The WR stars and the Of/WN star form the luminous tip in the diagram. We used the bolometric corrections of Smith and Maeder (1989) for the WR stars and Massey et al. (1989) for the OB stars.

We note the coexistence of a WN3, WN7 stars and an Of/WN6 star in an association which apparently is very young. In addition, we find the luminosities of all these stars to be very similar. These factors indicate that there may be an evolutionary link between these stars. Presently we can however not exclude the possibility that one or several WR stars are not physical members of the association. MGWR 4 indeed is strongly reddened (Morgan \& Good 1987) and might be a background star. More detailed results are in preparation and will be presented elsewhere (Lortet et al. 1990).

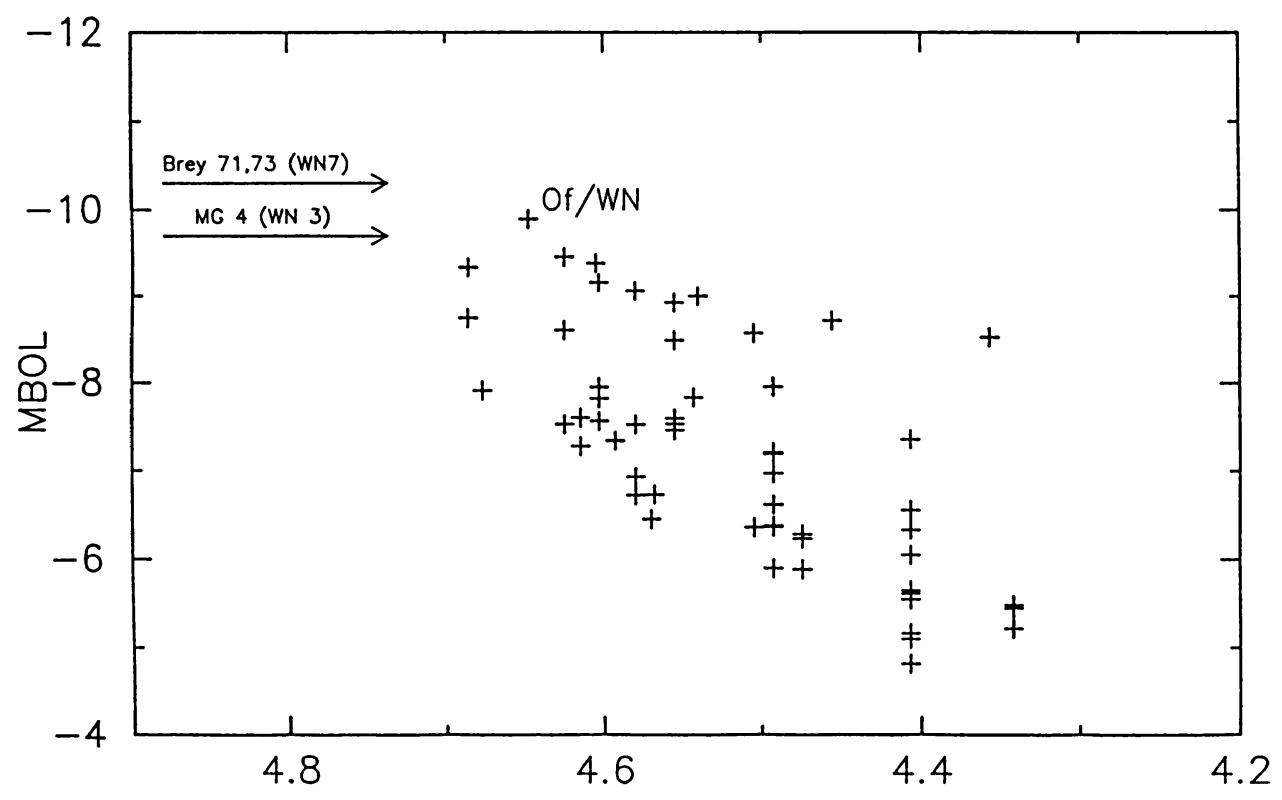

Fig. 2: HR diagram $M_{\text {bol }}$ versus $\log \left(T_{\text {eff }}\right)$ of the OB association LH 99. The luminosity of the WR stars is indicated by arrows.

\subsection{LH 90}

The central part of $\mathrm{LH} 90$ is shown in Fig 1 where also the positions of the WR stars are indicated. The earliest stars in LH 90 are of 05 spectral type. All $O$ stars are located in the western and central part of the association. The six WR stars now known in LH 
90 are also situated in the same area. The WR population consists of four WN5 to WN7 stars, a WC4 and a WN3 star. Three of the WR stars are located in tight stellar clusters (Fig 1).

The cluster located in the center of the association (labelled $\beta$ by Lortet and Testor 1984 ) is particularly interesting because the three visually brightest objects in it are a WN7, a WN3 and an O3If/WN6 star (Testor and Schild 1990). Spectra of these objects are shown in Fig 3. The cluster stars are very likely coeval and physiscal members.

Fig. 3: Spectra of the three visually brightest stars in the central tight cluster in LH 90 . For identification see Fig. 1.

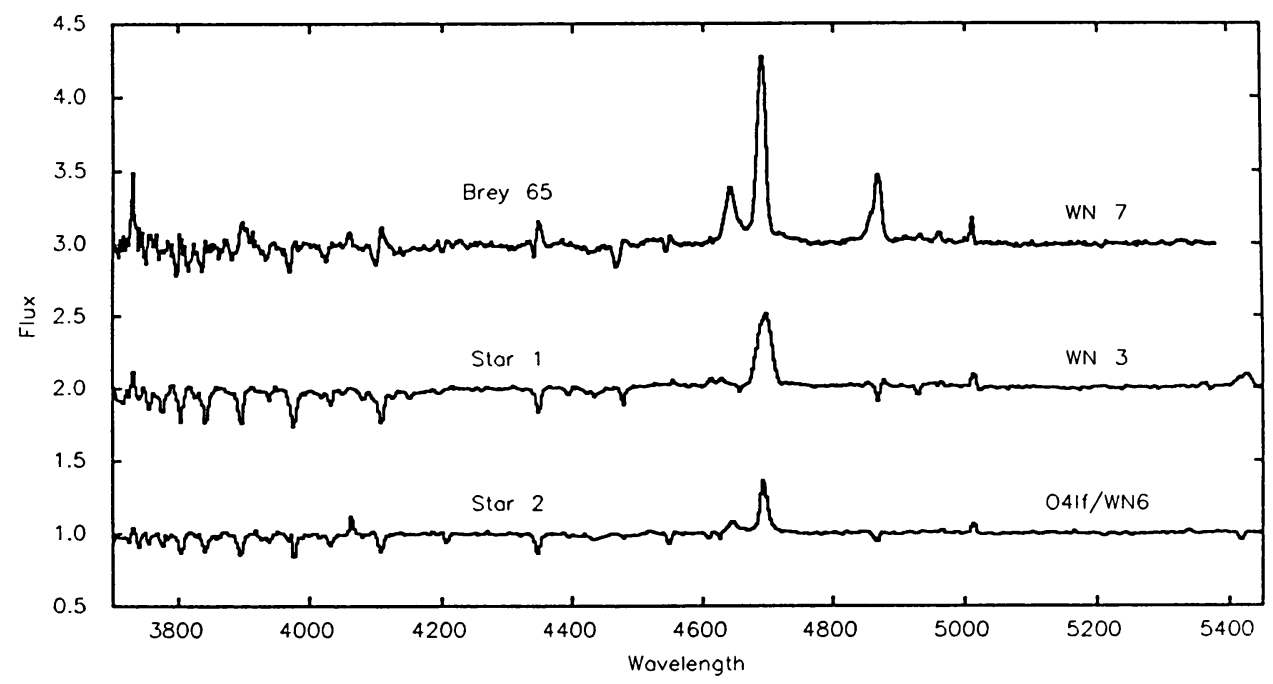

\subsection{LH 104}

Our photometric observations cover the association almost completely but the spectroscopic observations were centered on an area of 3' $\times 2.4^{\prime}$ which contains the three WR stars Brey 94, 95 and MGWR 6 . We thus obtained spectra of 60 stars located in the southern half of the association as defined by Lucke (1972).

The earliest stars in the surveyed part of LH 104 are of 05 and 06.5 spectral type. It therefore seems to be of a similar age to or slightly older than LH 90. A more detailed description of this association and its environment can be found in Lortet and Testor (1988).

\subsection{LH 89}

We concentrated the spectroscopic observations on the area around the WR stars Brey 60, 61 and 64 which are of WN3, WN4 and WN9-10 spectral type, respectively. We obtained spectra of more than 60 surrounding stars and the earliest stars turned out to be of 09 spectral type. The most luminous stars in this association have $M_{b o l} \approx-9$ which matches well the luminosities of the three WR stars (Fig. 4). LH 89 is the oldest of the associations surveyed here. The WR/O ratio is 1.5 which roughly is an order of magnitude larger than in $\mathrm{LH} 99$. 
Fig. 4:HR diagram of LH 89. The luminosities of the WR stars are indicated by arrows.

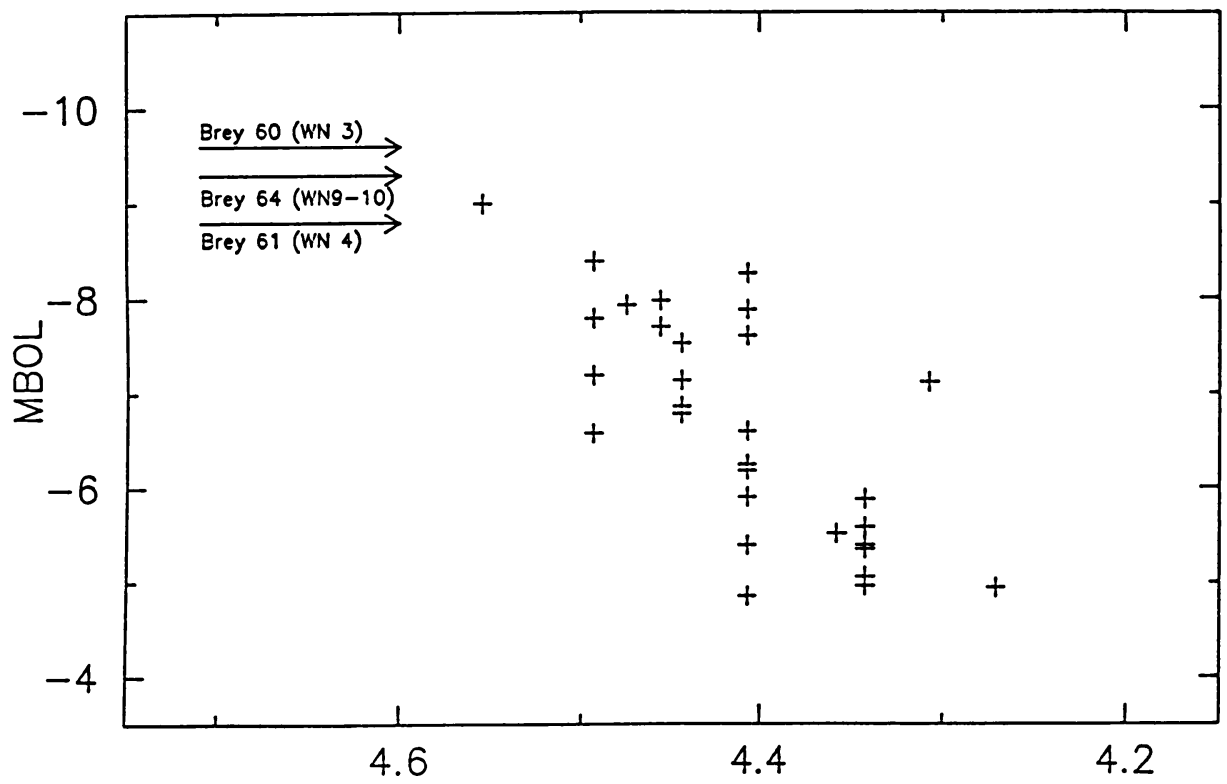

\section{Discussion}

In Table 1, the surveyed $\mathrm{OB}$ associations are ordered according to the earliest stars present. An increasing age sequence from $\mathrm{LH} 99$, to LH 90, LH 104 and LH 89 is thereby indicated. The presence of very hot stars is also reflected by HII emission as seen e.g. on $H \alpha$ images: $\mathrm{LH} 99$ is completely embedded in strong nebular emission; LH 90 and LH 104 show only moderate central nebular emission but are surrounded by strong filaments. The central parts of these associations seem to have already been swept clear by stellar winds and SNe. LH 89 finally is practically free from gaseous emission.

Table 1. Hot star population in the surveyed OB associations. Spectral types of earliest stars, slash stars and WR stars as well as the WR/O number ratios are given.

\begin{tabular}{llclc}
\hline & earliest stars & /stars & WR stars & WR/O \\
LH 99 & O3V,03-4V,03-4III & O3If/WN6 & WN7, WN7, WN3-4 & 0.16 \\
LH 90 & O4-5V, O5V, O5I & O4If/WN6 & WN7,WN7, WN6,WN5-6 & 0.40 \\
& & & WC4, WN3 & \\
LH 104 & O5I, O6.5V, O8V & - & WN4, WC5, WC5 & 0.50 \\
LH 89 & O9V, O9.5V, B0V & - & WN3, WN4, WN9-10 & 1.5 \\
\hline
\end{tabular}


There is also a marked increase in the WR/O ratio from LH 99 to LH 89. As seen in LH 89, it is possible that under special conditions the number of WR stars can be of the same order or even exceed the number of $O$ stars. This also indicates that WR progenitors can have initial masses at least as low as late 0 stars.

The subtype distribution of the WR stars also shows some remarkable age preferences with the exception of WN3 and WN4 stars which seem to occur in clusters of all ages. The WN7, WN6 and WN5 stars are confined to the two youngest associations whereas the WC4 and WC5 stars occur in the medium age associations. The WN9-10 star finally occurs in the oldest association. This is in agreement with a previous study on another WN9-10 star (Brey 18) which is located in an even older association (Schild 1987).

The occurence of WR stars in the four associations surveyed suggests the following evolutionary connections between $O$ and WR stars in the LMC:

$$
\begin{aligned}
& \text { early } \mathrm{O} \rightarrow \text { OIf/WN6 } \rightarrow \text { WN7 } \rightarrow \text { WN3? } \\
& \text { mid } \mathrm{O} \rightarrow \text { WN6-4 } \rightarrow \text { WC4,5 } \\
& \text { late } \mathrm{O} \rightarrow \text { WN3,4 or WN9-10 }
\end{aligned}
$$

The hottest stars present in a stellar association only provide a lower age limit for the aggregate. There may however be a considerable age spread in these associations. We are planning to examine this age dispersion with colour-magnitude diagrams in the near future. In relation to the evolutionary status of WR stars, a possible age spread is however not of crucial importance because as it can be seen in Figs 2 and 4, the WR stars usually are among the most luminous stars in an association. This implies that they originate from the most massive and therefore youngest subpopulation present in the cluster.

\section{References}

Breysacher, J.: 1981, Astron. Astrophys. Suppl. 43, 203

Breysacher, J.: 1986, Astron. Astrophys. 160, 185

Fehrenbach, C., Duflot, M., Acker, A.: 1976, Astron. Astrophys. Suppl. 33, 115

Humphreys, R.M., Nichols, M., Massey, P.: 1985, Astron. J. 90, 101

Lortet, M.C., Testor, G.: 1984, Astron. Astrophys. 139, 330

Lortet, M.C., Testor, G.: 1988, Astron. Astrophys. 194, 11

Lortet, M.C., Schild, H., Testor, G.: 1990, in preparation

Lucke, P.B.: 1972, Ph.D. Thesis, Univ. Washington

Massey, P., Parker, J.W., Garmany, C.D.: 1989, Astron. J. 98, 1305

Moffat, A.F.J.: 1989, Astrophys. J. 347, 373

Morgan, D.H., Good, A.R.: 1987, Mon. Not. R. astr. Soc. 224, 435

Schild, H.: 1987, Astron. Astrophys. 173, 405

Schild, H., Maeder, A.: 1984, Astron. Astrophys. 136, 237

Smith, L.F.: 1968, Mon. Not. R. astr. Soc. 140, 409

Smith, L.F., Maeder, A.: 1989, Astron. Astrophys. 211, 71

Testor, G., Schild, H.: 1990, Astron. Astrophys, accepted

Torres-Dodgen, A.V., Massey, P.: 1988, Astron. J. 96, 1076

Walborn, N.R.: 1977, Astrophys. J. 215, 53

Walborn, N.R.: 1986, in IAU Symp. 116, 185 